\title{
First Subject In Identity
}

National Cancer Institute

\section{Source}

National Cancer Institute. First Subject In Identity. NCI Thesaurus. Code C142559.

The name of the first individual who is enrolled into a clinical study. 